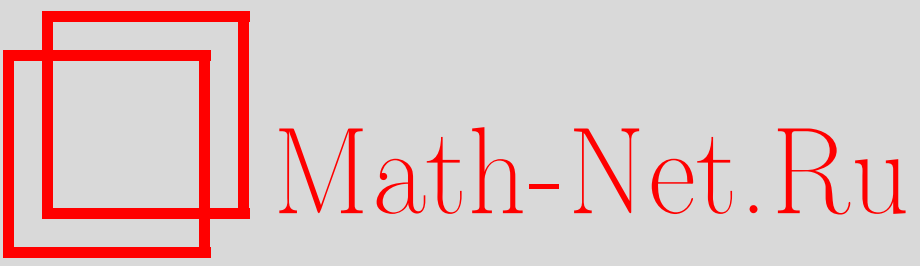

М. Д. Миссаров, Непрерывный предел в фермионной иерархической модели, ТМФ, 1999, том 118, номер 1, 40-50

DOI: https://doi.org/10.4213/tmf684

Использование Общероссийского математического портала Math-Net.Ru подразумевает, что вы прочитали и согласны с пользовательским соглашением

http://www.mathnet.ru/rus/agreement

Параметры загрузки:

IP: 3.89 .197 .203

26 апреля 2023 г., 15:59:51 
ТЕОРЕТИЧЕСКАЯ

И МАТЕМАТИЧЕСКАЯ

ФИЗИКА

Том 118, № 1

январь, 1999

(C) 1999 г.

М. Д. Миссаров*

\title{
НЕПРЕРЫВНЫЙ ПРЕДЕЛ В ФЕРМИОННОЙ ИЕРАРХИЧЕСКОЙ МОДЕЛИ
}

\begin{abstract}
Обсуждается проблема строгого построения непрерывного предела в фермионной иерархической модели. Непрерывный предел строится как предел полей на измельчающихся иерархических решетках и является полем на $p$-адическом континууме. Исследуется вопрос о восстановлении констант связи непрерывной модели по константам связи ее дискретизации.
\end{abstract}

Как уже отмечалось ранее [1-2], p-адические модели теории поля являются естественным непрерывным обобщением иерархических моделей Дайсона [3-6]. p-Адические модели возникают в связи с широким спектром проблем математической физики $[7,8]$.

В этой статье мы исследуем проблему построения непрерывного предела в рамках так называемой иерархической фермионной модели (см. [9-11]). Соответствующая проблема в рамках обычных решетчатых моделей квантовой теории поля является основной (и трудной) задачей конструктивной теории поля $[12,13]$. Как известно, в этих моделях гауссовская часть фиксирована (например, задается решетчатым аналогом оператора Лапласа). Существование непрерывного предела означает, что имеются пределы корреляционных функций, когда шаг решетки стремится к нулю. При этом константы связи промежуточных решетчатых моделей зависят от шага решетки и стремятся к бесконечности, если обрезание снимается.

Особенностью нашей модели является то, что гауссовская часть задается действием

$$
H_{0}\left(\psi^{*} ; \alpha\right)=c(\alpha) \int|x-y|^{-\alpha d}\left(\bar{\psi}_{1}(x) \psi_{1}(y)+\bar{\psi}_{2}(x) \psi_{2}(y)\right) d x d y
$$

где $x$ и $y-d$-мерные $p$-адические аргументы, $d x$ - соответствующая мера Хаара, $\bar{\psi}^{*}(x)=\left(\bar{\psi}_{1}(x), \psi_{1}(x), \bar{\psi}_{2}(x), \psi_{2}(x)\right)$ - 4-компонентное грассманово-значное поле (компоненты являются образующими алгебры Грассмана), $|\cdot|-p$-адическая норма, $c(\alpha)$ - некоторая нормируюшая константа, $\alpha$ - вешественный параметр. Этот гамильтониан задает так называемое автомодельное (скейлинг-инвариантное) фермионное поле с параметром автомодельности $\alpha$.

* Казанский государственный университет, Казань, Россия. E-mail: Moukadas.Missarov@ksu.ru 
Значение $\alpha=1+2 / d$ задает $p$-адический аналог гамильтониана, который в вешественном случае задается оператором Лапласа. Заметим только, что в отличие от вешественного случая при $\alpha=1+2 / d p$-адический вариант описывает модель с дальнодействием.

Негауссовская часть задается гамильтонианом

$$
H_{1}=r \int\left(\bar{\psi}_{1}(x) \psi_{1}(x)+\bar{\psi}_{2}(x) \psi_{2}(x)\right) d x+g \int \bar{\psi}_{1}(x) \psi_{1}(x) \bar{\psi}_{2}(x) \psi_{2}(x) d x
$$

Нашу модель можно считать фермионным аналогом иерархической $\varphi^{4}$-модели, своей негауссовской частью она имитирует модель Гросса-Невё $[14,15]$. Отметим также, что некоторый вырожденньй вариант фермионной иерархической модели рассматривался в работе [16].

Мы изучаем дискретный (иерархический) вариант гамильтониана $H_{0}+H_{1}$. Гауссовская часть иерархической модели инвариантна относительно преобразования блок-спиновой ренормализационной группы (РГ) Каданова-Вильсона с параметром $\alpha$. Действие РГ в пространстве констант связи вычисляется явно [9], и обратное отображение также существует. Это обстоятельство является основным упрощающим моментом в проблеме построения непрерывного предела в нашей модели.

В частности, показано, что для рассмотренных в [10] значений $\alpha, r, g$, для которых сушествует термодинамический предел в иерархической модели, сушествует предел корреляционных функций, когда шаг иерархической решетки стремится к нулю. Область сушествования термодинамического предела в плоскости $(r, g)$, как следует из результатов [10] и компьютерных экспериментов, составляет множество полной меры в $R^{2}$, если $\alpha>1$.

Новым является то, что при $\alpha>2$ сушествует предел констант связи решетчатых теорий поля, когда шаг решетки стремится к нулю. Этот предел и задает непрерьвную теорию поля, дискретизация которой приводит к исходной иерархической модели.

Пусть $T$ - отображение, которое сопоставляет константам связи $r$ и $g$ исходной иерархической модели константы связи предельной непрерывной теории. Тогда при $\alpha>2$ отображение $T$ удовлетворяет коммутационному соотношению

$$
T R=S T,
$$

где $R$ - преобразование иерархической РГ в плоскости $(r, g), S$ - диагональная матрица, заданная собственными числами дифференциала отображения $R$ в нуле. Отсюда видно, что отображение $T$ обратно к нормализуюшему преобразованию $P$, которое определяется функциональным уравнением

$$
R P=P S .
$$

Отображение $P$ можно трактовать как оператор дискретизации, который сопоставляет константам связи непрерывного поля константы связи дискретизованного поля (подробнее см. [17]). 
Итак, $T=P^{-1}$. Однако, как известно из общей теории нормальных форм [18], отображение $P\left(\right.$ и $\left.P^{-1}\right)$ может быть определено не только для $\alpha>2$, но и для нерезонансных значений $\alpha<2$ (более точное утверждение см. ниже) и задается сходяшимся рядом по степеням $r$ и $g$ в некоторой окрестности нуля. Таким образом, отображение $T$ может быть доопределено и в области $1<\alpha<2$, за исключением некоторой дискретной серии значений $\alpha$, и задает константы связи непрерывной теории, отвечающей исходной иерархической модели. Однако эти константы связи не могут быть определены прямым предельным процессом, тем более не могут быть определены при резонансных значениях $\alpha$.

Возможно, аналогичная картина справедлива и для бозонных моделей в вещественном случае, если задавать ядро гауссовской части степенью оператора Лапласа. Сам же оператор Лапласа отвечает резонансному значению.

Отметим, что серия резонансных значений $\alpha$ для функционального уравнения (2) совпадает с серией ультрафиолетовых полюсов $p$-адических фейнмановских амплитуд непрерывного варианта модели.

Напомним некоторые определения [9]. Пусть $Q_{p}$ - поле $p$-адических чисел, $|\cdot|_{p}-$ $p$-адическая норма на $Q_{p}$. Обозначим через $\{x\}$ дробную часть $p$-адического числа $x=$ $c_{-n} p^{-n}+\cdots+c_{-1} p^{-1}+c_{0}+c_{1} p+\ldots$ :

$$
\{x\}=c_{-n} p^{-n}+\cdots+c_{-1} p^{-1} .
$$

Для $x=\left(x_{1}, \ldots, x_{d}\right) \in Q_{p}^{d}$ положим

$$
|x|_{p}=\max _{i}\left|x_{i}\right|_{p}, \quad\{x\}=\left(\left\{x_{1}\right\}, \ldots,\left\{x_{d}\right\}\right) .
$$

Тогда дискретное множество

$$
T_{p}^{d}=\left\{x \in Q_{p}^{d}: x=\{x\}\right\}
$$

можно рассматривать как иерархическую решетку с размером элементарной ячейки $n=p^{d}$, где иерархическое расстояние $d(i, j)=|i-j|_{p}, i, j \in T_{p}^{d}$.

Рассмотрим 4-компонентное фермионное поле $\psi^{*}(x)=\left(\bar{\psi}_{1}(x), \psi_{1}(x), \bar{\psi}_{2}(x), \psi_{2}(x)\right)$, $x \in Q_{p}^{d}$. Группа скейлинговых преобразований определяется как

$$
\left(S_{\lambda}(\alpha) \psi^{*}\right)(x)=|\lambda|^{\left(1-\frac{\alpha}{2}\right) d} \psi^{*}(\lambda x)
$$

где $\lambda \in Q_{p}, \alpha$-вешественный параметр. Тогда гауссовское состояние, инвариантное относительно скейлинговой группы, задается гамильтонианом (1), в котором мы выбираем нормирующую константу

$$
c(\alpha)=\frac{f_{n}(\alpha)}{f_{n}(1-\alpha)}, \quad f_{n}(\alpha)=\left(1-n^{-\alpha}\right)^{-1}, \quad n=p^{d}
$$


Определим дискретизацию поля $\psi^{*}$ на решетку $T_{p}^{d}$ как поле $\xi^{*}$ :

$$
\xi^{*}(j)=\int \psi^{*}(j+x) \chi(x) d x, \quad j \in T_{p}^{d},
$$

где $\chi(x)$ - характеристическая функция шара $Z_{p}^{d}=\left\{x:|x|_{p} \leqslant 1\right\}$. Тогда дискретизация преобразования $S_{\lambda}(\alpha), \lambda=p^{-1} \in Q_{p}$, есть обычное иерархическое блок-спиновое РГ-преобразование:

$$
\left(r(\alpha) \xi^{*}\right)(j)=n^{-\alpha / 2} \sum_{i \in B(j)} \xi^{*}(j), \quad B(j)=\left\{i \in T_{p}^{d}:|p i-j| \leqslant 1\right\} .
$$

Дискретизация гауссовского поля с гамильтонианом (1) будет задаваться гамильтонианом

$$
H_{0}^{\prime}\left(\xi^{*} ; \alpha\right)=\sum_{i, j} h(i, j ; \alpha)\left(\bar{\xi}_{1}(i) \xi_{1}(j)+\bar{\xi}_{2}(i) \xi_{2}(j)\right)
$$

где

$$
h(i, j ; \alpha)=\frac{f_{n}(\alpha)}{f_{n}(1-\alpha)}\left(1-\delta_{i j}\right)|i-j|^{-\alpha d}+\frac{f_{n}(\alpha)}{f_{n}(1)} \delta_{i j} .
$$

Рассмотрим теперь, как предлагалось выше, формальное фермионное поле с гамильтонианом

$$
H\left(\psi^{*} ; \alpha ; r, g\right)=H_{0}\left(\psi^{*} ; \alpha\right)+\int L\left(\psi^{*}(x) ; r, g\right) d x,
$$

где

$$
L\left(\psi^{*}(x) ; r, g\right)=r\left(\bar{\psi}_{1}(x) \psi_{1}(x)+\bar{\psi}_{2}(x) \psi_{2}(x)\right)+g \bar{\psi}_{1}(x) \psi_{1}(x) \bar{\psi}_{2}(x) \psi_{2}(x) .
$$

Заметим сразу, что скейлинговое преобразование $S_{\lambda}(\alpha), \lambda=p^{-1} \in Q_{p}$, действует в пространстве гамильтонианов (3) линейно: $H\left(\psi^{*} ; \alpha ; r, g\right) \rightarrow H\left(\psi^{*} ; \alpha ; n^{\alpha-1} r, n^{2 \alpha-3} g\right)$. Обозначим линейное преобразование в плоскости констант связи $(r, g) \rightarrow\left(n^{\alpha-1} r\right.$, $n^{2 \alpha-3} g$ ) через $S(\alpha)$.

Дискретизация поля $\psi^{*}(x)$ с гамильтонианом (3) будет задаваться гамильтонианом

$$
H^{\prime}\left(\xi^{*} ; \alpha ; r, g\right)=H_{0}^{\prime}(\xi ; \alpha)-\sum_{j} \ln \left\langle\exp \left\{-\int L\left(\xi^{*}(j)+\eta^{*}(x) ; r, g\right) d x\right\}\right\rangle_{\mu\left(d \eta^{*}\right)}
$$

где усреднение берется по гауссовскому полю $\eta^{*}(x)$ с носителем в шаре $Z_{p}^{d}$ с нулевым средним и бинарной корреляционной функцией

$$
\begin{aligned}
& \left\langle\bar{\eta}_{k}(x) \eta_{l}(y)\right\rangle=\delta_{k l}\left(\frac{f_{n}(2-\alpha)}{f_{n}(\alpha-1)}|x-y|^{(\alpha-2) d}-\frac{f_{n}(2-\alpha)}{f_{n}(1)}\right), \\
& \left\langle\bar{\eta}_{k}(x) \bar{\eta}_{l}(y)\right\rangle=\left\langle\eta_{k}(x) \eta_{l}(y)\right\rangle=0, \quad x, y \in Z_{p}^{d}, \quad k, l=1,2 .
\end{aligned}
$$

В [17] показано, что функциональный интеграл

$$
\ln \left\langle\exp \left\{-\int L\left(\xi^{*}(i)+\eta^{*}(x) ; r, g\right) d x\right\}\right\rangle_{\mu\left(d \eta^{*}\right)}
$$


может быть представлен в виде $L\left(\xi^{*}(i) ; u(r, g), v(r, g)\right)$, где отображение $(r, g) \rightarrow$ $(u(r, g), v(r, g))$ (обозначим его через $P(\alpha))$ является нормализуюшим преобразованием к отображению РГ $R(\alpha)$ в нуле:

$$
R(\alpha) P(\alpha)=P(\alpha) S(\alpha) .
$$

Напомним, что действие РГ-преобразования в пространстве констант связи $(r, g)$ вычисляется явно [9]:

$$
\begin{gathered}
R(\alpha)(r, g)=\left(r^{\prime}, g^{\prime}\right), \\
r^{\prime}=n^{\alpha-1}\left(\frac{(r+1)^{2}-g}{(r+1)^{2}-\frac{g}{n}}(r+1)-1\right), \\
g^{\prime}=n^{2 \alpha-3}\left(\frac{(r+1)^{2}-g}{(r+1)^{2}-\frac{g}{n}}\right)^{2} g .
\end{gathered}
$$

Заметим, что отображение $S(\alpha)$ задается диагональной матрицей, собственные числа которой являются собственными числами дифференциала РГ-преобразования $R(\alpha)$ в нуле.

В случае, если $\alpha>3 / 2$, собственные числа $\lambda_{1}(\alpha)=n^{\alpha-1}>1$ и $\lambda_{2}(\alpha)=n^{2 \alpha-3}>1$, мы попадаем в область действия классической теоремы Пуанкаре [18]. По этой теореме для отображения $P(\alpha)$ существует разложение в сходящийся при достаточно малых $r, g$ ряд по степеням $r, g$ при условии, что $\alpha$ не является резонансным значением. Напомним, что $\alpha$ называется нерезонансным значением, если

$$
\lambda_{1}(\alpha) \neq \lambda_{1}^{m_{1}}(\alpha) \lambda_{2}^{m_{2}}(\alpha), \quad \lambda_{2}(\alpha) \neq \lambda_{1}^{m_{1}}(\alpha) \lambda_{2}^{m_{2}}(\alpha),
$$

где $m_{1}$ и $m_{2}-$ неотрицательные целые такие, что $m_{1}+m_{2} \geqslant 2$. Если $1 \leqslant \alpha \leqslant 3 / 2$ и $\lambda_{1}(\alpha)>1, \lambda_{2}(\alpha)<1$, мы попадаем в так называемую область Зигеля. В этом случае для сходимости отображения $P(\alpha)$ необходимо, помимо условия нерезонансности, чтобы $\alpha$ плохо приближалось рациональными числами [18].

Для $P(\alpha)$ вьполнено условие нормировки. Если мы обозначим

$$
P(\alpha)\left(\begin{array}{l}
r \\
g
\end{array}\right)=\left(\begin{array}{c}
u(r, g) \\
v(r, g)
\end{array}\right)
$$

то сходяшиеся в окрестности нуля ряды $u(r, g)$ и $v(r, g)$ удовлетворяют условию

$$
\left.\frac{\partial u(r, g)}{\partial r}\right|_{r=0, g=0}=1,\left.\quad \frac{\partial v(r, g)}{\partial g}\right|_{r=0, g=0}=1 .
$$

Обратимся теперь к проблеме построения непрерывного предела. Пусть $\xi^{*}$ - поле на решетке $T_{p}^{d}$, заданное гамильтонианом

$$
H^{\prime}\left(\xi^{*} ; \alpha ; r, g\right)=H_{0}^{\prime}(\xi ; \alpha)+\sum_{j \in T_{p}^{d}} L\left(\xi^{*}(j) ; r, g\right),
$$


и пусть $(r, g)$ принадлежит к тем областям плоскости, для которых доказано сушествование термодинамического предела в данной иерархической модели в работе [10].

Обозначим через $\xi_{m}^{*}$ поле на решетке $T_{p}^{d}$ с гамильтонианом

$$
H^{\prime}\left(\xi_{m}^{*} ; \alpha ; r^{(-m)}, g^{(-m)}\right), \quad\left(\begin{array}{c}
r^{(-m)} \\
g^{(-m)}
\end{array}\right)=R^{-m}\left(\begin{array}{l}
r \\
g
\end{array}\right), \quad m=0,1,2, \ldots
$$

Рассмотрим последовательность все более мелких иерархических решеток $T_{p, m}^{d}=$ $p^{m} T_{p}^{d}, m=0,1, \ldots$ Определим последовательность полей $\zeta_{m}^{*}$, заданных на решетках $T_{p, m}^{d}$, соотношениями

$$
\zeta_{m}^{*}(j)=p^{d m\left(1-\frac{\alpha}{2}\right)} \xi_{m}^{*}\left(p^{-m} j\right), \quad j \in T_{p, m}^{d}
$$

Таким образом, гамильтониан поля $\zeta_{m}^{*}(j)$ имеет вид

$$
\begin{aligned}
H_{m}^{\prime}\left(\zeta_{m}^{*}\right)= & \sum_{i, j \in T_{p}^{d}} h(i, j ; \alpha)\left[p ^ { - 2 d m ( 1 - \alpha / 2 ) } \left(\bar{\zeta}_{m, 1}\left(p^{m} i\right) \zeta_{m, 1}\left(p^{m} j\right)+\right.\right. \\
& \left.\left.+\bar{\zeta}_{m, 2}\left(p^{m} i\right) \zeta_{m, 2}\left(p^{m} j\right)\right)\right]+\sum_{i \in T_{p}^{d}} L\left(p^{-d m(1-\alpha / 2)} \zeta_{m}^{*}\left(p^{m} i\right) ; r, g\right)= \\
= & H_{m, 0}^{\prime}\left(\zeta_{m}^{*} ; \alpha\right)+\sum_{i \in T_{p, m}^{d}} p^{-m d} L\left(\zeta_{m}^{*}(i) ; \lambda_{1}^{m} r^{(-m)}, \lambda_{2}^{m} g^{(-m)}\right),
\end{aligned}
$$

где гауссовская часть

$$
\begin{aligned}
H_{m, 0}^{\prime}\left(\zeta_{m}^{*} ; \alpha\right)= & \sum_{\substack{i, j \in T_{p, m}^{d}, i \neq j}}|i-j|^{-\alpha d} p^{-2 m d}\left(\bar{\zeta}_{m, 1}(i) \zeta_{m, 1}(j)+\bar{\zeta}_{m, 2}(i) \zeta_{m, 2}(j)\right)+ \\
& +\sum_{i \in T_{p, m}^{d}} p^{m d(\alpha-2)} \frac{f_{n}(\alpha)}{f_{n}(1)}\left(\bar{\zeta}_{m, 1}(i) \zeta_{m, 1}(i)+\bar{\zeta}_{m, 2}(i) \zeta_{m, 2}(i)\right)
\end{aligned}
$$

Обозначим гамильтониан, задаваемьй правой частью формулы $(6)$, через $H_{m}^{\prime}\left(\zeta_{m}^{*} ; \alpha\right.$; $\left.\lambda_{1}^{m} r^{(-m)}, \lambda_{2}^{m} g^{(-m)}\right)$. Заметим, что гауссовская часть $H_{m, 0}^{\prime}\left(\zeta^{*} ; \alpha\right)$ является гамильтонианом дискретизации непрерывного гауссовского поля на решетку $T_{p, m}^{d}$ и весь гамильтониан $H_{m}^{\prime}$ записан в виде дискретной аппроксимации на решетке $T_{p, m}^{d}$ гамильтониана непрерывного поля.

Так как поля $\xi_{m}^{*}$ связаны между собой преобразованием РГ:

$$
p^{-\frac{\alpha d}{2}} \sum_{|p j-i| \leqslant 1} \xi_{m}^{*}(j)=\xi_{m-1}^{*}(i)
$$

то мы можем отсюда видеть, что поля $\zeta_{m}^{*}$ связаны между собой оператором усреднения

$$
p^{-d} \sum_{|p j-i| \leqslant 1} \zeta_{m}^{*}\left(p^{m} j\right)=\zeta_{m-1}^{*}\left(p^{m-1} i\right), \quad i, j \in T_{p}^{d}
$$


Таким образом, мы можем трактовать поля $\zeta_{m}^{*}$ как дискретизацию непрерьвного поля $\psi^{*}$ на решетку $T_{p, m}^{d}$ :

$$
\zeta_{m}^{*}(i)=\int \psi^{*}(x) \chi\left(\frac{x-i}{p^{m}}\right) d x, \quad i \in T_{p, m}^{d}
$$

где $\chi(x)$ - характеристическая функция шара $Z_{p}^{d}$.

Напомним $[7,19]$, что естественным пространством основных функций на $Q_{p}^{d}$ является пространство локально-постоянных финитных функций $D\left(Q_{p}^{d}\right)$. При этом последовательность $f_{i}(x) \in D\left(Q_{p}^{d}\right), i=1,2, \ldots$, стремится к нулю, если:

1 ) все функции $f_{i}(x)$ равны нулю вне некоторого (не зависящего от $i$ ) компактного множества;

2) существует такое целое положительное $n$, что $f_{i}(x)=f_{i}(y)$, если $|x-y| \leqslant p^{-n}$, $i=1,2, \ldots$;

3 ) последовательность $f_{i}(x)$ стремится к нулю при $i \rightarrow \infty$ равномерно по $x$.

Мы будем говорить, что непрерывное поле $\psi^{*}$ на $Q_{p}^{d}$ задается состоянием $\rho$ над пространством обобщенных функций $D^{\prime}\left(Q_{p}^{d}\right)$, если определены корреляционные функции

$$
\rho\left(\psi_{k_{1}}\left(f_{1}\right) \bar{\psi}_{l_{1}}\left(g_{1}\right) \ldots \psi_{k_{s}}\left(f_{s}\right) \bar{\psi}_{l_{s}}\left(g_{s}\right)\right)
$$

где $f_{1}, g_{1}, \ldots, f_{s}, g_{s} \in D\left(Q_{p}^{d}\right), k_{i}, l_{i}=1,2, i=1, \ldots, s$.

Поскольку функции $f_{1}, \ldots, g_{s}$ можно представить как конечную сумму индикаторов $p$-адических шаров достаточно малого диаметра, корреляционные функции (8) будут определены, если будут определены корреляционные функции всех полей $\zeta_{m}^{*}$, которые заданы состояниями $\tilde{\rho}_{m}$, задаваемыми гамильтонианами $H_{m}^{\prime}\left(\zeta_{m}^{*}\right)$ :

$$
\begin{aligned}
\tilde{\rho}_{m}\left(\zeta_{m, k_{1}}\left(i_{1}\right) \bar{\zeta}_{m, l_{1}}\left(j_{1}\right) \ldots \zeta_{m, k_{s}}\left(i_{s}\right) \bar{\zeta}_{m, l_{s}}\left(j_{s}\right)\right)= \\
=\frac{\left\langle\zeta_{m, k_{1}}\left(i_{1}\right) \bar{\zeta}_{m, l_{1}}\left(j_{1}\right) \ldots \zeta_{m, k_{s}}\left(i_{s}\right) \bar{\zeta}_{m, l_{s}}\left(j_{s}\right) \exp \left\{-H_{m}^{\prime}\left(\zeta_{m}^{*}\right)\right\}\right\rangle_{0}}{\left\langle\exp \left\{-H_{m}^{\prime}\left(\zeta_{m}^{*}\right)\right\}\right\rangle_{0}} .
\end{aligned}
$$

Здесь среднее $\langle\cdot\rangle_{0}$ понимается как интеграл по бесконечному числу антикоммутирующих переменных, сидящих в узлах решетки $T_{p, m}^{d}$, и корректно определено только в случае, если существует термодинамический предел. Однако заметим, что в силу соотношения (5) видно, что

$$
\begin{aligned}
& \tilde{\rho}_{m}\left(\zeta_{m, k_{1}}\left(i_{1}\right) \bar{\zeta}_{m, l_{1}}\left(j_{1}\right) \ldots \zeta_{m, k_{s}}\left(i_{s}\right) \bar{\zeta}_{m, l_{s}}\left(j_{s}\right)\right)= \\
& =p^{d m(2-\alpha) s} \frac{\left\langle\xi_{m, k_{1}}^{*}\left(p^{-m} i_{1}\right) \ldots \bar{\xi}_{m, l_{s}}^{*}\left(p^{-m} j_{s}\right) \exp \left\{-H^{\prime}\left(\xi_{m}^{*} ; \alpha ; r^{(-m)}, g^{(-m)}\right)\right\}\right\rangle_{0}}{\left\langle\exp \left\{-H^{\prime}\left(\xi_{m}^{*} ; \alpha ; r^{(-m)}, g^{(-m)}\right)\right\}\right\rangle_{0}} .
\end{aligned}
$$

Если термодинамический предел для поля $\xi^{*} \equiv \xi_{0}^{*}$ на решетке $T_{p}^{d}$, заданного гамильтонианом $H^{\prime}\left(\xi_{0}^{*} ; \alpha ; r, g\right)$, сушествует, то то же самое верно и для поля $\xi_{m}^{*}$, заданного гамильтонианом $H^{\prime}\left(\xi_{m}^{*} ; \alpha ; r^{(-m)}, g^{(-m)}\right)$, поскольку [10] сушествование термодинамического предела определяется асимптотическими свойствами последовательности $\left(r^{(-m+N)}, g^{(-m+N)}\right)$ при $N \rightarrow \infty$.

Таким образом, если сводить проблему построения непрерывного поля к построению корреляционных функций, то будет доказана следуюшая теорема. 
ТеОрема 1. Для всех точек плоскости констант связи $(r, g)$, для которых существует термодинамический предел в иерархической фермионной модели, существует непрерывный предел с корреляционными функциями непрерывного поля, определенными пределами формулой (9).

Отметим еще раз, что область значений констант связи, для которой доказано существование термодинамического предела, непуста (подробнее см. [10]).

Поскольку гамильтонианы $H_{m}^{\prime}\left(\zeta_{m}^{*} ; \alpha ; \lambda_{1}^{m} r^{(-m)}, \lambda_{2}^{m} g^{(-m)}\right)$ можно рассматривать как дискретные аппроксимации гамильтониана непрерывного поля $H\left(\psi^{*} ; \alpha ; \lambda_{1}^{m} r^{(-m)}\right.$, $\left.\lambda_{2}^{m} g^{(-m)}\right)$, возникает естественный вопрос, существует ли предел

$$
\lim _{m \rightarrow \infty}\left(\begin{array}{l}
\lambda_{1}^{m} r^{(-m)} \\
\lambda_{2}^{m} g^{(-m)}
\end{array}\right)=\lim _{m \rightarrow \infty} S^{m} R^{-m}\left(\begin{array}{l}
r \\
g
\end{array}\right),
$$

и если да, то как этот предел связан с константами связи начального дискретного поля $(r, g)$. Пусть $U$ обозначает множество всех точек $(r, g)$, которые при итерациях обратного отображения $R^{-1}$ стремятся к тривиальной неподвижной точке $(0,0)$. Докажем следуюшую теорему.

Теорема 2. Пусть $\alpha>2$. Тогда для всех $(r, g) \in U$ предел

$$
\lim _{m \rightarrow \infty} S^{m} R^{-m}\left(\begin{array}{l}
r \\
g
\end{array}\right)=T\left(\begin{array}{l}
r \\
g
\end{array}\right)
$$

существует и отображение Т удовлетворяет коммутачионному соотношению

$$
T R=S T
$$

ДокАЗАТЕЛЬСТво. Отображение $R^{-1}$ может быть вычислено явно:

$$
R^{-1}\left(\begin{array}{l}
r \\
g
\end{array}\right)=\left(\begin{array}{c}
\lambda_{1}^{-1}\left(\frac{\left(r+\lambda_{1}\right)^{2}-g}{\left(r+\lambda_{1}\right)^{2}-n g}\left(r+\lambda_{1}\right)-\lambda_{1}\right) \\
\lambda_{2}^{-1}\left(\frac{\left(r+\lambda_{1}\right)^{2}-g}{\left(r+\lambda_{1}\right)^{2}-n g}\right)^{2} g
\end{array}\right) .
$$

В окрестности нуля это отображение имеет разложение в сходяшийся ряд по степеням $r$ и $g$ :

$$
R^{-1}\left(\begin{array}{c}
r \\
g
\end{array}\right)=\left(\begin{array}{c}
\lambda_{1}^{-1} r+\frac{n-1}{\lambda_{1}^{2}} g+t_{1}(r, g) \\
\lambda_{2}^{-1} g+t_{2}(r, g)
\end{array}\right),
$$

где $t_{1}(r, g)$ и $t_{2}(r, g)$ - ряды по степеням $r$ и $g$ без свободных и линейных членов. Сделаем замену переменных

$$
\left(\begin{array}{l}
x_{1} \\
x_{2}
\end{array}\right)=A\left(\begin{array}{l}
r \\
g
\end{array}\right), \quad A=\left(\begin{array}{cc}
1 & \frac{(n-1) \lambda_{2}}{\lambda_{1}\left(\lambda_{2}-\lambda_{1}\right)} \\
0 & 1
\end{array}\right) .
$$

Тогда дифференциал отображения

$$
R_{1}=A R A^{-1}
$$


задается диагональной матрицей $S$, и мы получаем, что

$$
S^{m} R^{-m}=S^{m} A R_{1}^{-m} A^{-1}=S^{m} A S^{-m} S^{m} R_{1}^{-m} A^{-1} .
$$

Заметим, что

$$
S^{m} A S^{-m}=\left(\begin{array}{ll}
1 & \left(\frac{\lambda_{1}}{\lambda_{2}}\right)^{m} \\
0 & \frac{(n-1) \lambda_{2}}{\lambda_{1}\left(\lambda_{2}-\lambda_{1}\right)} \\
1
\end{array}\right),
$$

и если $\alpha>2$, то т.к. $\lambda_{1}(\alpha)=n^{\alpha-1}<n^{2 \alpha-3}=\lambda_{2}(\alpha)$, мы получаем

$$
\lim _{m \rightarrow \infty} S^{m} A S^{-m}=\left(\begin{array}{ll}
1 & 0 \\
0 & 1
\end{array}\right) .
$$

Мы имеем разложение для $R_{1}^{-1}$ в нуле:

$$
R_{1}^{-1}\left(\begin{array}{c}
x_{1} \\
x_{2}
\end{array}\right)=\left(\begin{array}{c}
\lambda_{1}^{-1} x_{1}+t_{1}^{\prime}\left(x_{1}, x_{2}\right) \\
\lambda_{2}^{-1} x_{2}+t_{2}^{\prime}\left(x_{1}, x_{2}\right)
\end{array}\right) .
$$

Ряды $t_{1}^{\prime}\left(x_{1}, x_{2}\right)$ и $t_{2}^{\prime}\left(x_{1}, x_{2}\right)$ начинаются с мономов второго порядка. Пусть $|x|_{1}$ обозначает норму в $R^{2}$, определяемую как

$$
|x|_{1}=\max \left(\left|x_{1}\right|,\left|x_{2}\right|\right), \quad x=\left(\begin{array}{l}
x_{1} \\
x_{2}
\end{array}\right) .
$$

Пусть $U_{\varepsilon}=\left\{x \in R^{2}:|x|_{1}<\varepsilon\right\}$. В силу того что $\lambda_{2}^{-1}<\lambda_{1}^{-1}$ при $\alpha>2$, при достаточно малых $\varepsilon$ справедлива оценка

$$
\left|R_{1}^{-1}(x)\right|_{1}<\left(\lambda_{1}^{-1}+c_{1} \varepsilon\right)|x|_{1}
$$

где $c_{1}$ - некоторая константа, не зависящая от $\alpha$ и $\varepsilon$. Сушествует такое $\varepsilon_{0}$, что отображение $R_{1}^{-1}$ является сжимающим в $U_{\varepsilon_{0}}:\left|R_{1}^{-1}(x)\right|_{1}<\delta|x|_{1}, x \in U_{\varepsilon}, \delta<1$. Пусть

$$
V=\left\{x \in R^{2}: \lim _{m \rightarrow \infty} R_{1}^{-m}(x)=0\right\} .
$$

Ясно, что $V$ содержит окрестность нуля. Мы можем написать

$$
\lim _{m \rightarrow \infty} S^{m} R_{1}^{-m}(x)=S^{m_{0}} \lim _{m \rightarrow \infty} S^{m} R_{1}^{-m}\left(R_{1}^{-m_{0}}(x)\right)
$$

и для любого $x \in V$ существует $m_{0}$ такое, что $\left|R_{1}^{-m_{0}} x\right|_{1}<\varepsilon_{1}$ для любого наперед заданного $\varepsilon_{1}$. Выберем $\varepsilon_{1}$ так, чтобы

$$
\left|R_{1}^{-1}(x)-S^{-1} x\right|_{1} \leqslant c_{2}|x|_{1}^{2}, \quad\left|R_{1}^{-1}(x)\right|_{1}<\delta_{1}|x|_{1},
$$

где $c_{2}<\infty, \lambda_{1}^{-1}<\delta_{1}<1, \delta_{1}^{2}<\lambda_{2}^{-1}$ (последнее неравенство возможно в силу оценки (11) и того, что $\left.\lambda_{1}^{-2}=n^{2-2 \alpha}<n^{3-2 \alpha}=\lambda_{2}^{-1}\right)$. Тогда мы можем написать, что

$$
\begin{aligned}
& \left|S^{m+1} R_{1}^{-(m+1)}(x)-S^{m} R_{1}^{-m}(x)\right|_{1} \leqslant \\
& \quad \leqslant \lambda_{2}^{m+1}\left|R_{1}^{-1}\left(R_{1}^{-m}(x)\right)-S^{-1} R_{1}^{-m}(x)\right| \leqslant c_{2} \lambda_{2}^{m+1} \delta_{1}^{2 m}|x|_{1}^{2} .
\end{aligned}
$$


Поскольку $\left(\lambda_{2} \delta_{1}^{2}\right)^{m} \rightarrow 0$ при $m \rightarrow \infty$, последовательность $S^{m} R^{-m}(x)$ является фундаментальной и предел $\lim _{m \rightarrow \infty} S^{m} R^{-m}(x)$ сушествует для всех $x \in V$. Так как множество $U$ при замене (10) переходит в множество $V$, то первое утверждение теоремы доказано. Второе утверждение следует из очевидного равенства

$$
T=\lim _{m \rightarrow \infty} S^{m} R^{-m}=S \lim _{m \rightarrow \infty} S^{m-1} R^{-(m-1)} R^{-1}=S T R^{-1} .
$$

Заметим также, что поскольку отображение $T$ является равномерным пределом аналитических в некоторой окрестности нуля отображений $S^{m} R^{-m}$, то $T$ аналитично в нуле, и т.к.

$$
\left.\frac{\partial\left(S^{m} R^{-m}(r, g)\right)}{\partial r}\right|_{r=0, g=0}=1,\left.\quad \frac{\partial\left(S^{m} R^{-m}(r, g)\right)}{\partial g}\right|_{r=0, g=0}=1
$$

то

$$
\left.\frac{\partial T(r, g)}{\partial r}\right|_{r=0, g=0}=1,\left.\quad \frac{\partial T(r, g)}{\partial g}\right|_{r=0, g=0}=1
$$

Отсюда и из (4) следует, что отображение $T$ является обратным к $P: T=P^{-1}$. Как уже отмечалось, отображение $P^{-1}$ определено для почти всех значений $\alpha<2$. В области $3 / 2<\alpha<2$ резонансные значения образуют дискретную серию

$$
\alpha_{k}=\frac{3}{2}+\frac{1}{2(2 k-1)}, \quad k=1,2, \ldots
$$

Для всех $\alpha$, которые отличны от $\alpha_{k}, k=1,2, \ldots$, отображение $P^{-1}$ имеет сходяшееся разложение в окрестности нуля. В области $1<\alpha<3 / 2$ любое рациональное число является резонансным. Для всех остальных $\alpha$ отображение $P^{-1}$ определено при условии, что пара $\lambda_{1}(\alpha), \lambda_{2}(\alpha)$ является набором $(C, v)$-типа, т.е.

$$
\left|\lambda_{1}-\lambda_{1}^{m_{1}} \lambda_{2}^{m_{2}}\right| \geqslant C|m|^{-v}, \quad\left|\lambda_{2}-\lambda_{1}^{m_{1}} \lambda_{2}^{m_{2}}\right| \geqslant C|m|^{-v},
$$

$m=m_{1}+m_{2} \geqslant 2, m_{1} \geqslant 0, m_{2} \geqslant 0$. Это завершает доказательство теоремы.

Благодарности. Работа выполнена при финансовой поддержке Российского фонда фундаментальных исследований (грант № 97-01-00375). 


\section{Список литературы}

[1] Э. Ю. Лернер, М. Д. Миссаров. ТМФ. 1989. Т. 78. № 2. С. 248.

[2] E. Yu. Lerner, M. D. Missarov. Commun. Math. Phys. 1989. V. 121. P. 35.

[3] P. M. Bleher, Ya. G. Sinai. Commun. Math. Phys. 1973. V. 33. P. 23.

[4] P. Collet, J.-P. Eckmann. A renormalization group analysis of the hierarchical model in statistical mechanics. Berlin-Heidelberg-New York: Springer, 1978.

[5] Я. Г. Синай. Теория фазовых переходов: Строгие результаты. М.: Наука, 1980.

[6] P. M. Bleher, P. Major. Ann. Prob. 1987. V. 15. P. 431.

[7] В.С. Владимиров, И. В. Волович, Е. И. Зеленов. р-Адический анализ и математическая физика. М.: Наука, 1994.

[8] L. Brekke, P. G. O. Freund. Phys. Rep. 1993. V. 233. P. 1.

[9] E. Yu. Lerner, M. D. Missarov. J. Stat. Phys. 1994. V. 76. № 3/4. P. 805.

[10] Э. Ю. Лернер, М. Д. Миссаров. ТМФ. 1996. Т. 107. № 2. С. 201.

[11] М. Д. Миссаров. ТМФ. 1998. Т. 114. № 3. С. 323.

[12] Джс. Глимм, А. Джаффе. Математические методы квантовой физики. М.: Мир, 1984.

[13] K. Gawedski, A. Kupiainen. Commun. Math. Phys. 1985. V. 99. P. 197.

[14] K. Gawedski, A. Kupiainen. Commun. Math. Phys. 1985. V. 102. P. 1.

[15] J. Feldman, J. Magnen, V. Rivasseu, R. Seneor. Commun. Math. Phys. 1986. V. 103. P. 67.

[16] T. C. Dorlas. Commun. Math. Phys. 1991. V. 136. P. 169.

[17] M. D. Missarov. Lett. Math. Phys. 1994. V. 32. P. 347.

[18] В. И. Арнольд. Дополнительные главы теории обыкновенных дифференциальных уравнений. М.: Наука, 1978.

[19] И. М. Гельфанд, М. И. Граев, И. И. Пятецкий-Шапиро. Теория представлений и автоморфные функции. М.: Наука, 1966.

Поступила в редакцию 29.V.1998 г. 